\title{
Subclass of Analytic Functions Related with Pascal Distribution Series
}

\author{
Amnah E. Shammaky $\mathbb{D}^{\mathrm{D}},{ }^{1}$ Basem Aref Frasin $\mathbb{D D}^{2}{ }^{2}$ and Tamer M. Seoudy $\mathbb{D}^{3,4}$ \\ ${ }^{1}$ Faculty of Science, Jazan University, Jazan, Saudi Arabia \\ ${ }^{2}$ Department of Mathematics, Faculty of Science, Al Al-Bayt University, Mafraq, Jordan \\ ${ }^{3}$ Department of Mathematics, Jamoum University College, Umm Al-Qura University, Makkah, Saudi Arabia \\ ${ }^{4}$ Department of Mathematics, Faculty of Science, Fayoum University, Fayoum 63514, Egypt
}

Correspondence should be addressed to Basem Aref Frasin; bafrasin@yahoo.com

Received 24 August 2021; Accepted 16 February 2022; Published 4 March 2022

Academic Editor: Ji Gao

Copyright (c) 2022 Amnah E. Shammaky et al. This is an open access article distributed under the Creative Commons Attribution License, which permits unrestricted use, distribution, and reproduction in any medium, provided the original work is properly cited.

The prime purpose of this article is to derive a necessary and sufficient condition for a linear operator associated with the Pascal distribution series to be in the class $\mathfrak{I} \mathcal{S}(\mu, \sigma, \delta)$ of analytic functions. Moreover, inclusion relation and an integral operator linked to the Pascal distribution series is considered. We have also provided some results as corollaries of our theorems.

\section{Introduction and Class Definition}

Let $\mathbb{C}$ be the set of all complex numbers. We denote by $\mathfrak{G}$, the set of all analytic functions in the unit disk $\Delta=\{\xi \in \mathbb{C}:|\xi|<1\}$ that have the series of the form.

$$
f(\xi)=\xi+\sum_{n=2}^{\infty} a_{n} \xi^{n}
$$

For functions $f \in \mathbb{G}$ given by (1) and $g \in \mathbb{G}$ given by $g(\xi)=\xi+\sum_{n=2}^{\infty} b_{n} \xi^{n}$, we recall that the well-known Hadamard product of $f$ and $g$ is given by.

$$
f(\xi) * g(\xi):=\xi+\sum_{n=2}^{\infty} a_{n} b_{n} \xi^{n}, \quad \xi \in \Delta .
$$

For $\varepsilon \in \mathbb{C}-\{0\}$ and $-1 \leq \mathfrak{B}<\mathfrak{A} \leq 1$, we say that a function $f \in \mathfrak{G}$ lies in the class $\mathscr{R}^{\varepsilon}(\mathfrak{A}, \mathfrak{B})$ if

$$
\left|\frac{f^{\prime}(\xi)-1}{(\mathfrak{A}-\mathfrak{B}) \mathcal{E}-\mathfrak{B}\left[f^{\prime}(\xi)-1\right]}\right|<1, \quad \xi \in \Delta .
$$

The function class $\mathscr{R}^{\varepsilon}(\mathfrak{A}, \mathfrak{B})$ was introduced in [1]. In [2], Magesh and Prameela defined the following class:
Definition 1. A function $f \in G$ lies in the class $\delta(\mu, \sigma, \delta)$ if the following inequality is satisfied.

$$
\begin{aligned}
& \Re\left\{\frac{\xi f^{\prime}(\xi)+\mu(2 \mu-1) \xi^{2} f^{\prime \prime}(\xi)}{4 \mu(1-\mu) \xi+\mu(2 \mu-1) \xi f^{\prime}(\xi)+\left(2 \mu^{2}-3 \mu+1\right) f(\xi)}-\sigma\right\} \\
& >\delta\left|\frac{\xi f^{\prime}(\xi)+\mu(2 \mu-1) \xi^{2} f^{\prime \prime}(\xi)}{4 \mu(1-\mu) \xi+\mu(2 \mu-1) \xi f^{\prime}(\xi)+\left(2 \mu^{2}-3 \mu+1\right) f(\xi)}-1\right|,
\end{aligned}
$$

where $\delta \geq 0,-1 \leq \sigma<1,0 \leq \mu \leq 1$, and $\xi \in \Delta$.

We also let

$$
\mathfrak{I} \mathcal{S}(\mu, \sigma, \delta)=\mathcal{S}(\mu, \sigma, \delta) \cap \mathfrak{T},
$$

where $\mathfrak{T}$ is be a subclass of $\mathfrak{G}$ consisting of functions of the form

$$
f(\xi)=\xi-\sum_{n=2}^{\infty}\left|a_{n}\right| \xi^{n}, \quad(\xi \in \Delta) .
$$

We note that, by specializing the parameters $\mu, \sigma$ and $\delta$, we obtain the following subclasses studied by various authors. 
(1) $\mathfrak{I} \mathcal{S}(0, \sigma, 0)=\mathfrak{T}(\sigma)$ and $\mathfrak{I} \mathcal{S}(1, \sigma, 0)=\mathscr{K}(\sigma)$ (Silverman [3])

(2) $\mathfrak{I} \mathcal{S}((1 / 2), \sigma, 0)=\mathscr{P}(\sigma)$ (Al-Amiri [4], Gupta and Jain [5] and Sarangi and Uralegaddi [6])

(3) $\mathfrak{I} \mathcal{S}((1 / 2), \sigma, \delta)=\mathfrak{I} \mathscr{R}(\sigma, \delta)$ (Rosy [7] and Stephen and Subramanian [8])

(4) $\mathfrak{T} \mathcal{S}(0, \sigma, \delta)=\mathfrak{T} \mathcal{S}(\sigma, \delta)$ $\mathfrak{T} \mathcal{S}(1, \sigma, \delta)=\mathscr{U} \mathscr{C} \mathscr{V}(\sigma, \delta)$ (Bharati et al. [9])

(5) $\mathfrak{I} \mathcal{S}(0,0, \delta)=\mathfrak{T} \mathcal{S}_{p}(\delta)$ (Subramanian et al. [10])

(6) $\mathfrak{I} \mathcal{S}(1,0, \delta)=\mathscr{U} \mathscr{C} \mathscr{V}(\delta)$ (Subramanian et al. [11])

In [12], El-Deeb et al. provided a power series expansion whose coefficients are related to the probabilities of the Pascal distribution.

$$
\Phi_{r}^{t}(\xi)=\xi+\sum_{n=2}^{\infty}\left(\begin{array}{c}
n+t-2 \\
t-1
\end{array}\right) r^{n-1}(1-r)^{t} \xi^{n}, \quad \xi \in \Delta,
$$

where $t \geq 1 ; 0 \leq r \leq 1$. We also define the series

$$
\begin{array}{r}
\Upsilon_{r}^{t}(\xi)=2 \xi-\Phi_{r}^{t}(\xi)=\xi-\sum_{n=2}^{\infty}\left(\begin{array}{c}
n+t-2 \\
t-1
\end{array}\right) r^{n-1}(1-r)^{t} \xi^{n}, \\
\xi \in \Delta .
\end{array}
$$

Next, let us consider the linear operator

$$
\mathfrak{\Im}_{r}^{t} f: \mathfrak{G} \longrightarrow \mathfrak{G},
$$

defined by means of the convolution or Hadamard product.

$$
\begin{aligned}
\mathfrak{\Im}_{r}^{t} f(\xi) & =\Phi_{r}^{t}(\xi) * f(\xi) \\
& =\xi+\sum_{n=2}^{\infty}\left(\begin{array}{c}
n+t-2 \\
t-1
\end{array}\right) r^{n-1}(1-r)^{t} a_{n} \xi^{n}, \quad \xi \in \Delta .
\end{aligned}
$$

Several results on the engagements between different subclasses of analytic univalent functions and special functions or distribution series have been studied in the literature (see for example, [13-27]). Herein, we provide a necessary and sufficient condition for $\Upsilon_{r}^{t}(\xi)$ to be in the aforementioned class $\mathfrak{T} \mathcal{S}(\mu, \sigma, \delta)$ and investigate an inclusion property of the class $\mathfrak{I} \mathcal{S}(\mu, \sigma, \delta)$ associated with the operator $\mathfrak{\Im}_{r}^{t} f$. Ultimately, we provide conditions for the integral operator $\mathscr{G}_{r}^{t} f(\xi)=\int_{0}^{\xi} \Upsilon_{r}^{t}(t) / t \mathrm{~d} t$ to be in the investigated class $\mathfrak{I} \mathcal{S}(\mu, \sigma, \delta)$.

We shall need the following lemmas to state and prove our results.

Lemma 1 (see [2]). Given that $\delta \geq 0,-1 \leq \sigma<1$ and $(1 / 2) \leq \mu<1$. A function $f \in \mathfrak{I}$ belongs to the class $\mathfrak{I} \mathcal{S}(\mu, \sigma, \delta)$ if and only if the following inequality is satisfied.

$$
\sum_{n=2}^{\infty}\left[\mathrm{F}(\mu, \delta) n^{2}+\Theta(\mu, \sigma, \delta) n+\Lambda(\mu, \sigma, \delta)\right]\left|a_{n}\right| \leq 1-\sigma
$$

where

$$
\begin{aligned}
\mathrm{F}(\mu, \delta) & =\left(2 \mu^{2}-\mu\right)(1+\delta), \\
\Theta(\mu, \sigma, \delta) & =\left(\mu-2 \mu^{2}\right)(1+\sigma+2 \delta)+1+\delta, \\
\Lambda(\mu, \sigma, \delta) & =\left(3 \mu-1-2 \mu^{2}\right)(\sigma+\delta) .
\end{aligned}
$$

The result is sharp for the function.

$$
f(\xi)=\xi-\frac{1-\sigma}{\mathrm{F}(\mu, \delta) n^{2}+\Theta(\mu, \sigma, \delta) n+\Lambda(\mu, \sigma, \delta)} \xi^{n} .
$$

Lemma 2 (see [1]). If $f \in \mathbb{G}$ lies in the class $\mathscr{R}^{\mathcal{E}}(\mathfrak{A}, \mathfrak{B})$, then

$$
\left|a_{n}\right| \leq(\mathfrak{A}-\mathfrak{B}) \frac{|\mathcal{E}|}{n}, \quad n \in \mathbb{N}-\{1\} .
$$

The result is sharp.

\section{The Necessary and Sufficient Condition}

Throughout this paper, we use significantly the following identities for $0 \leq r<1$ and $t \geq 1$.

$$
\begin{aligned}
\sum_{n=2}^{\infty}\left(\begin{array}{c}
n+t-2 \\
t-1
\end{array}\right) r^{n-1} & =\frac{1}{(1-r)^{t}}-1, \\
\sum_{n=2}^{\infty}(n-1)\left(\begin{array}{c}
n+t-2 \\
t-1
\end{array}\right) r^{n-1} & =\frac{r t}{(1-r)^{t+1}}, \\
\sum_{n=3}^{\infty}(n-1)(n-2)\left(\begin{array}{c}
n+t-2 \\
t-1
\end{array}\right) r^{n-1} & =\frac{r^{2} t(t+1)}{(1-r)^{t+2} .}
\end{aligned}
$$

Using Lemma 1, we get the following necessary and sufficient condition for $\Upsilon_{r}^{t}(\xi)$ to be in the investigated class $\mathfrak{I} \mathcal{S}(\mu, \sigma, \delta)$.

Theorem 1. For $t \geq 1, \Upsilon_{r}^{t}(\xi) \in \mathfrak{T} \mathcal{S}(\mu, \sigma, \delta)$ if and only if

$$
\begin{aligned}
& \frac{\mathrm{F}(\mu, \delta) r^{2} t(t+1)}{(1-r)^{2}}+(3 \mathrm{~F}(\mu, \delta)+\Theta(\mu, \sigma, \delta)) \frac{r t}{1-r} \\
& +(\mathrm{F}(\mu, \delta)+\Theta(\mu, \sigma, \delta)+\Lambda(\mu, \sigma, \delta))\left(1-(1-r)^{t}\right) \leq 1-\sigma .
\end{aligned}
$$

Proof. From Lemma 1, we only need to show that

$$
\begin{aligned}
& \sum_{n=2}^{\infty}\left[\mathrm{F}(\mu, \delta) n^{2}+\Theta(\mu, \sigma, \delta) n+\Lambda(\mu, \sigma, \delta)\right] \\
& \left(\begin{array}{c}
n+t-2 \\
t-1
\end{array}\right) r^{n-1}(1-r)^{t} \leq 1-\sigma .
\end{aligned}
$$

Putting $n=(n-1)+1$ and $n^{2}=(n-1)(n-2)+3$ $(n-1)+1$, in $(17)$, we have 


$$
\begin{aligned}
& \sum_{n=2}^{\infty}\left[\mathrm{F}(\mu, \delta) n^{2}+\Theta(\mu, \sigma, \delta) n+\Lambda(\mu, \sigma, \delta)\right]\left(\begin{array}{c}
n+t-2 \\
t-1
\end{array}\right) r^{n-1}(1-r)^{t} \\
= & {\left[\begin{array}{c}
\sum_{n=3}^{\infty} \mathrm{F}(\mu, \delta)(n-1)(n-2)\left(\begin{array}{c}
n+t-2 \\
t-1
\end{array}\right) r^{n-1} \\
+\sum_{n=2}^{\infty}[3 \mathrm{~F}(\mu, \delta)+\Theta(\mu, \sigma, \delta)](n-1)\left(\begin{array}{c}
n+t-2 \\
t-1
\end{array}\right) r^{n-1} \\
+\sum_{n=2}^{\infty}[\mathrm{F}(\mu, \delta)+\Theta(\mu, \sigma, \delta)+\Lambda(\mu, \sigma, \delta)]\left(\begin{array}{c}
n+t-2 \\
t-1
\end{array}\right) r^{n-1}
\end{array}\right] } \\
= & \frac{\mathrm{F}(\mu, \delta) r^{2} t(t+1)}{(1-r)^{2}+(3 \mathrm{~F}(\mu, \delta)+\Theta(\mu, \sigma, \delta))} \\
& \cdot \frac{r t}{1-r}+(\mathrm{F}(\mu, \delta)+\Theta(\mu, \sigma, \delta)+\Lambda(\mu, \sigma, \delta))\left(1-(1-r)^{t}\right) .
\end{aligned}
$$

But this last expression is bounded previously by $1-\sigma$ if and only if (16) is satisfied.

\section{Inclusion Properties}

Next, we show that $\mathfrak{\Im}_{r}^{t}\left(\mathscr{R}^{\mathcal{E}}(\mathfrak{A}, \mathfrak{B})\right) \subset \mathfrak{T} \mathcal{S}(\mu, \sigma, \delta)$.

Proof. According to Lemma 1, it suffices to prove that

Theorem 2. For $t>1$ and $f \in \mathscr{R}^{\varepsilon}(\mathfrak{A}, \mathfrak{B}), \mathfrak{\Im}_{r}^{t} f(\xi) \in \mathfrak{I} \mathcal{S}$ $(\mu, \sigma, \delta)$ if

$$
(\mathfrak{A}-\mathfrak{B})|\varepsilon|\left[\frac{\mathrm{F}(\mu, \delta) r t}{1-r}+(\mathrm{F}(\mu, \delta)+\Theta(\mu, \sigma, \delta))\left(\frac{1}{(1-r)^{t}}-1\right)+\frac{\Lambda(\mu, \sigma, \delta)}{r(t-1)}\left[(1-r)-(1-r)^{t}-r(t-1)(1-r)^{t}\right]\right] \leq 1-\sigma
$$

$$
\begin{aligned}
& \sum_{n=2}^{\infty}\left[\mathrm{F}(\mu, \delta) n^{2}+\Theta(\mu, \sigma, \delta) n+\Lambda(\mu, \sigma, \delta)\right] \\
& \left(\begin{array}{c}
n+t-2 \\
t-1
\end{array}\right) r^{n-1}(1-r)^{t}\left|a_{n}\right| \leq 1-\sigma .
\end{aligned}
$$

$$
\begin{aligned}
& \sum_{n=2}^{\infty}\left[\mathrm{F}(\mu, \delta) n^{2}+\Theta(\mu, \sigma, \delta) n+\Lambda(\mu, \sigma, \delta)\right]\left(\begin{array}{c}
n+t-2 \\
t-1
\end{array}\right) r^{n-1}(1-r)^{t}\left|a_{n}\right| \\
\leq & (\mathfrak{A}-\mathfrak{B})|\varepsilon|(1-r)^{t}\left[\sum_{n=2}^{\infty}\left[\mathrm{F}(\mu, \delta) n+\Theta(\mu, \sigma, \delta)+\frac{\Lambda(\mu, \sigma, \delta)}{n}\right]\left(\begin{array}{c}
n+t-2 \\
t-1
\end{array}\right) r^{n-1}\right]
\end{aligned}
$$




$$
\begin{aligned}
= & (\mathfrak{A}-\mathfrak{B})|\varepsilon|(1-r)^{t}\left[\begin{array}{c}
\left.\sum_{n=2}^{\infty}[\mathrm{F}(\mu, \delta)(n-1)+\mathrm{F}(\mu, \delta)+\Theta(\mu, \sigma, \delta)]\left(\begin{array}{c}
n+t-2 \\
t-1
\end{array}\right) r^{n-1}\right] \\
\sum_{n=2}^{\infty} \frac{\Lambda(\mu, \sigma, \delta)}{n}\left(\begin{array}{c}
n+t-2 \\
t-1
\end{array}\right) r^{n-1}
\end{array}\right] \\
= & (\mathfrak{A}-\mathfrak{B})|\varepsilon|\left[\frac{\mathrm{F}(\mu, \delta) r t}{1-r}+(\mathrm{F}(\mu, \delta)+\Theta(\mu, \sigma, \delta))\left(\frac{1}{(1-r)^{t}}-1\right)\right. \\
& \left.+\frac{\Lambda(\mu, \sigma, \delta)}{r(t-1)}\left[(1-r)-(1-r)^{t}-r(t-1)(1-r)^{t}\right]\right] .
\end{aligned}
$$

Thus, the proof is completed since the RHS of the above inequality is bounded by $1-\sigma$.

\section{An Integral Operator}

In this section, we will consider the following integral operator.

$$
\mathscr{G}_{r}^{t} f(\xi)=\int_{0}^{\xi} \frac{\Upsilon_{r}^{t}(t)}{t} \mathrm{~d} t
$$

Theorem 3. For $t>1, \mathscr{G}_{r}^{t} f(\xi) \in \mathfrak{I} \mathcal{S}(\mu, \sigma, \delta)$ if and only if

$$
\frac{\mathrm{F}(\mu, \delta) r t}{1-r}+(\mathrm{F}(\mu, \delta)+\Theta(\mu, \sigma, \delta))\left(\frac{1}{(1-r)^{t}}-1\right)+\frac{\Lambda(\mu, \sigma, \delta)}{r(t-1)}\left[(1-r)-(1-r)^{t}-r(t-1)(1-r)^{t}\right] \leq 1-\sigma
$$

Proof. From (8) and (22), we easily get

Clearly, we have

$$
\mathscr{G}_{r}^{t} f(\xi)=\xi-\sum_{n=2}^{\infty}\left(\begin{array}{c}
n+t-2 \\
t-1
\end{array}\right) r^{n-1}(1-r)^{t} \frac{\xi^{n}}{n}
$$

Then using Lemma 1, we only need to prove that.

$$
\begin{aligned}
& \sum_{n=2}^{\infty}\left[\mathrm{F}(\mu, \delta) n^{2}+\Theta(\mu, \sigma, \delta) n+\Lambda(\mu, \sigma, \delta)\right] \\
& \quad \times \frac{1}{n}\left(\begin{array}{c}
n+t-2 \\
t-1
\end{array}\right) r^{n-1}(1-r)^{t} \leq 1-\sigma .
\end{aligned}
$$

$$
\sum_{n=2}^{\infty}[\mathrm{F}(\mu, \delta) n+\Theta(\mu, \sigma, \delta)]\left(\begin{array}{c}
n+t-2 \\
t-1
\end{array}\right) r^{n-1}(1-r)^{t}+\sum_{n=2}^{\infty} \frac{\Lambda(\mu, \sigma, \delta)}{n}\left(\begin{array}{c}
n+t-2 \\
t-1
\end{array}\right) r^{n-1}(1-r)^{t} \leq 1-\sigma
$$

The rest of the proof can be made similar to that of Theorem 2 .

\section{Corollaries and Consequences}

Letting $\mu=1 / 2$ in Theorems $1-3$, one can get the following corollaries.

Corollary 1. For $t \geq 1, Y_{r}^{t}(\xi) \in \mathfrak{T} \mathscr{R}(\sigma, \delta)$ if and only if

$$
(1+\delta)\left[\frac{r t}{1-r}+\left(1-(1-r)^{t}\right)\right] \leq 1-\sigma
$$

Corollary 2. for $t>1$ and $f \in \mathscr{R}^{\varepsilon}(\mathfrak{A}, \mathfrak{B}), \mathfrak{\Im}_{r}^{t} f(\xi) \in$ $\mathfrak{T} \mathscr{R}(\sigma, \delta)$ if

$$
(\mathfrak{A}-\mathfrak{B})|\mathcal{\varepsilon}|\left[(1+\delta)\left(\frac{1}{(1-r)^{t}}-1\right)\right] \leq 1-\sigma .
$$


Corollary 3. For $t>1$, the integral operator $\mathscr{G}_{r}^{t} f(\xi)$ given by (22) is in the class $\mathfrak{T} \mathscr{R}(\sigma, \delta)$ if and only if

$$
(1+\delta)\left(\frac{1}{(1-r)^{t}}-1\right) \leq 1-\sigma .
$$

\section{Conclusions}

In the present paper and due the earlier works (see, for example, $[12,15,21])$, we find a necessary and sufficient condition and inclusion relation for Pascal distribution series to be in a class of analytic functions with negative coefficients. Furthermore, we consider an integral operator related to Pascal distribution series. Some interesting corollaries and applications of the results are also discussed.

\section{Data Availability}

No data were used to support this study.

\section{Conflicts of Interest}

The authors declare that they have no conflicts of interest.

\section{References}

[1] K. K. Dixit and S. K. Pal, "On a class of univalent functions related to complex order," Indian Journal of Pure and Applied Mathematics, vol. 26, no. 9, pp. 889-896, 1995.

[2] N. Magesh and V. Prameela, "Certain subclasses of uniformly convex functions and corresponding class of starlike functions," Malaya Journal of Matematik, vol. 1, no. 1, pp. 18-26, 2013.

[3] H. Silverman, "Univalent functions with negative coefficients," Proceedings of the American Mathematical Society, vol. 51, no. 1, 1975.

[4] H. S. Al-Amiri, "On a subclass of close-to-convex functions with negative coefficients," Mathematics, vol. 31, no. 54, pp. 1-7, 1989.

[5] V. P. Gupta and P. K. Jain, "Certain classes of univalent functions with negative coefficients II," Bulletin of the Australian Mathematical Society, vol. 15, no. 3, pp. 467-473, 1976.

[6] S. M. Sarangi and B. A. Uralegaddi, "The radius of convexity and starlikeness of certain classes of analytic functions with negative coefficients I, atti della accademia nazionale dei lincei," Classe di Scienze Fisiche, Matematiche e Naturali, vol. 65, no. 8, pp. 34-42, 1978.

[7] T. Rosy, Study on Subclasses of Univalent Functions, University of Madras, Chennai, India, 2002.

[8] B. A. Stephand and K. G. Subramanian, On a Subclass of Noshiro-Type Analytic Functions with Negative Coefficients, Ramanujan Mathematical Society, Tiruchirappalli, India, 1998.

[9] R. Bharati, R. Parvatham, and A. Swaminathan, "On subclasses of uniformly convex functions and corresponding class of starlike functions," Tamkang Journal of Mathematics, vol. 28, no. 1, pp. 17-32, 1997.

[10] K. G. Subramanian, T. V. Sudharsan, P. Balasubrahmanyam, and H. Silverman, "Class of uniformly starlike functions," Publicationes Mathematicae Debrecen, vol. 53, no. 4, pp. 309-315, 1998.
[11] K. G. Subramanian, G. Murugusundaramoorthy, P. Balasubrahmanyam, and H. Silverman, "Subclasses of uniformly convex and uniformly starlike functions," Mathematica Japonica, vol. 42, no. 3, pp. 517-522, 1995.

[12] S. M. El-Deeb, T. Bulboacă, and J. Dziok, "Pascal distribution series connected with certain subclasses of univalent functions," Kyungpook Mathematical Journal, vol. 59, pp. 301-314, 2019.

[13] M. Ahmad, B. Frasin, G. Murugusundaramoorthy, and A. Alkhazaleh, "An application of Mittag-Leffler-type poisson distribution on certain subclasses of analytic functions associated with conic domains," Heliyon, vol. 7, no. 10, Article ID e08109, 2021.

[14] M. K. Aouf, A. O. Mostafa, and H. M. Zayed, "Some constraints of hypergeometric functions to belong to certain subclasses of analytic functions," Journal of the Egyptian Mathematical Society, vol. 24, no. 3, pp. 361-366, 2016.

[15] T. Bulboacă and G. Murugusundaramoorthy, "Univalent functions with positive coefficients involving pascal distribution series," Communications of the Korean Mathematical Society, vol. 35, no. 3, pp. 867-877, 2020.

[16] N. E. Cho, S. Y. Woo, and S. Owa, "Uniform convexity properties for hypergeometric functions," Fractional Calculus and Applied Analysis, vol. 5, no. 3, pp. 303-313, 2002.

[17] H. E. Darwish, A. Y. Lashin, and E. M. Madar, "On subclasses of uniformly starlike and convex functions defined by struve functions," International Journal of Open Problems in Complex Analysis, vol. 8, no. 1, pp. 34-43, 2016.

[18] B. A. Frasin, "An application of generalized bessel functions on general class of analytic functions with negative coefficients," Applied Mathematics E-Notes, vol. 21, pp. 426-436, 2021.

[19] B. A. Frasin and I. Aldawish, "On subclasses of uniformly spirallike functions associated with generalized bessel functions," Journal of Function Spaces, vol. 2019, Article ID 1329462, 6 pages, 2019.

[20] B. A. Frasin, S. Porwal, and F. Yousef, "Subclasses of starlike and convex functions associated with Mittag-Leffler-type poisson distribution series," Montes Taurus Journal Pure and Applied Mathematics, vol. 3, no. 3, pp. 147-154, 2021.

[21] B. A. Frasin, S. R. Swamy, and A. K. Wanas, "Subclasses of starlike and convex functions associated with pascal distribution series," Kyungpook Mathematical Journal, vol. 61, pp. 99-110, 2021.

[22] B. A. Frasin, T. Al-Hawary, and F. Yousef, "Necessary and sufficient conditions for hypergeometric functions to be in a subclass of analytic functions," Afrika Matematika, vol. 30, 2018.

[23] N. Magesh, S. Porwal, and C. Abirami, "Starlike and convex properties for poisson distribution series," Studia Universitatis Babeş-Bolyai Mathematica, vol. 63, no. 1, pp. 71-78, 2018.

[24] E. P. Merkes and W. T. Scott, "Starlike hypergeometric functions," Proceedings of the American Mathematical Society, vol. 12, no. 6, pp. 885-888, 1961.

[25] G. Murugusundaramoorthy, "Application of pascal distribution series to ronning type starlike and convex functions," Advances in the Theory of Nonlinear Analysis and its Application.vol. 4, no. 4, pp. 243-251, 2020.

[26] S. Porwal, "An application of a poisson distribution series on certain analytic functions," Journal of Complex Analysis, vol. 2014, Article ID 984135, 3 pages, 2014.

[27] H. Silverman, "Starlike and convexity properties for hypergeometric functions," Journal of Mathematical Analysis and Applications, vol. 172, no. 2, pp. 574-581, 1993. 